\title{
Scheme for Improving Transmission Performance of Realtime Traffic in Handover Between HMIPv6 Intermap Domains
}

\author{
Wongil Park, Jonghyoun Choi, and Byunggi Kim* \\ Department of Computer Science, Soongsil University \\ prudent_woman@yahoo.co.kr, wide@sunny.ssu.ac.kr, \\ bgkimecomputing.ssu.ac.kr
}

\begin{abstract}
Many studies have been performed to improve the efficiency of mobile IP. Hierarchical MIPv6 (HMIPv6) was proposed due to the lack of MIPv6. The new protocol, that is, Mobility Anchor Point (MAP) receives all packets in place of Mobile Node (MN) and MAP services are transferred to Care of Address (CoA) of $\mathrm{MN}_{-}$. However, it can affect the whole network owing to concentration phase of registration occurred in hierarchical MAP structure. We propose the scheme that selects different MAP according to the traffic characteristic. The quantitative result and performance analysis presented in this paper show that our proposal can reduce the cost of location update by $5 \%$ and total cost of MN that moves frequently by $34 \%$.
\end{abstract}

\section{Introduction}

It is difficult for Mobile IPv6 (MIPv6) to support nodes with high mobility, because it is designed for low mobility devices $[1,5]$. Adding a new service area, a $\mathrm{MN}$ acquires a new address and informs the home agent (HA) of this new address through binding update message. The HA is located on its home network which might be far away from the current location. That is the reason why the connection setup delay and packet loss occur. Thus it influences end-to-end QoS of real-time traffic $[1,2]$.

Hierarchical MIPv6 (HMIPv6) was proposed to solve this problem. MAP is introduced to improve the binding problem of MIPv6 [3]. It acts as a temporary HA in the network.

In Fig. 1, we assume that a MN is associated with AR1. MAP will play a role of temporary HA. AR1 will be the local access router. The $\mathrm{MN}$ receives system information both from the MAP and from the local router. The MN generates a Regional Care of Address (RCoA) and an on-Link Care of Address (LCoA). The MN registers itself on the system using RCoA and LCoA. When lots of MNs are allocated to a single MAP in a hierarchical Mobile IP network, it might incur significant

\footnotetext{
* This work was supported by the Korea Research Foundation Grant (KRF-2004-005-D00198). 
signaling overhead and processing overload [4, 7]. To avoid these problems, MNs should be evenly distributed to all MAPs.

This paper proposes a load balancing scheme for a MAP in a hierarchical Mobile IP network. The structure of this paper is as follows. In section 2, we describe the mobility of MN in HMIPv6. Next, in section 3, we explains traffic characteristic of MN of MAP selection scheme proposed in this paper. In section 4, we describe the performance evaluation by numerical analysis. Finally, section 5 offers conclusion.

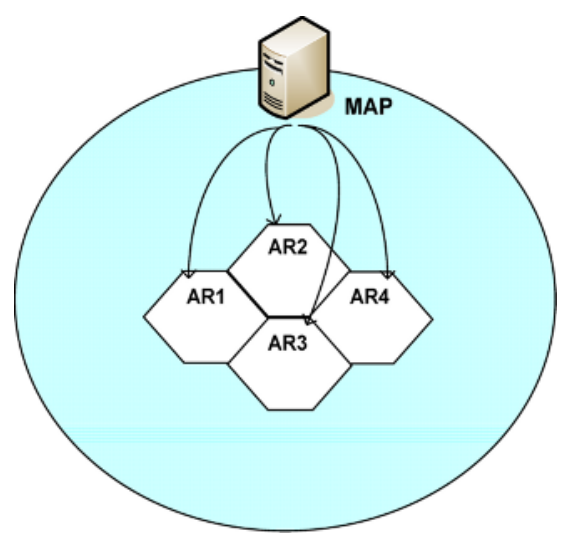

Fig. 1. HMIPv6 network structure

\section{Related Works}

There are several issues relating MAPs in a hierarchical Mobile IP. The first issue is which Access Router (AR)s would take roles of MAPs in a hierarchical network configuration. There are two options: one is to allow any AR in a hierarchical network to be a MAP and the other is to allow only a subset of ARs to be MAPs.

The second issue is on the load management of MAPs. It depends on the MAP configuration and the number of MNs. MAPs themselves which are responsible for mobility control of mobile terminals can be hierarchically configured. A decision algorithm is required on which MAP takes charge of each terminals. It is the problem of hierarchical configuration of MAPs and the access mechanism to them.

The third issue is how to relate mobility characteristic of MN to MAP selection.

For efficient mobility management of MNs, MAPs are introduced in HMIPv6. They act as local HAs. MAPs make it possible to provide better performance while minimizing modification of MIPv6 [6].

There are two kinds of handovers in HMIPv6 as shown in Fig. 2: micro handover and macro handover. In Fig. 2 micro handover occurs when a MN in the service area of AR1 of MAP2 enters the service area of AR3 of MAP2. Both AR1 and AR3 are under control of the same MAP2. Therefore the MN's MAP is not changed. 
When a MN moves from the service area of AR3 of MAP2 to that of AR1 of MAP3, a macro handover occurs. Its MAP is changed from MAP2 to MAP3. In case of micro handover, LCoA is changed but RCoA is not. So it needs to send a Binding Update (BU) message neither to the original HA nor to the Correspondent node $(\mathrm{CN})$. The MN still sends IP packets to MAP of $\mathrm{CN}$ and MAP forwards it to the correspondent.

However, macro handover accompanies MAP change. Therefore the BU message, which includes the new RCoA, must be sent to the original HA and the CN. It will increase handover delay. In this way MN's mobility has influence on handover performance and eventually on the configuration and selection of MAPs.

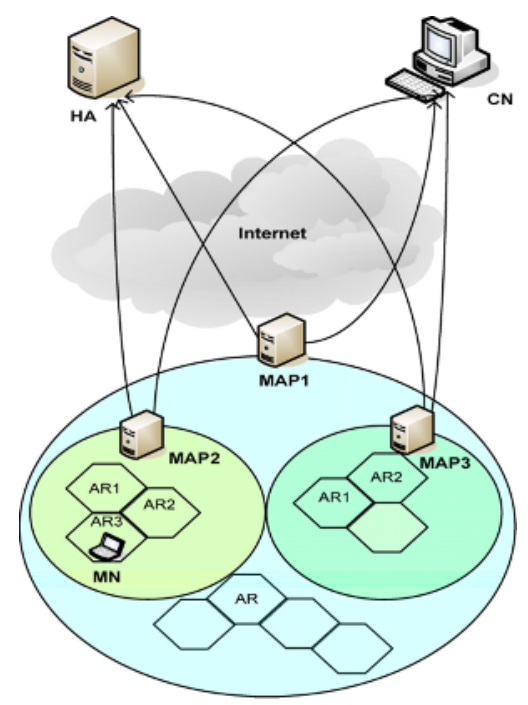

Fig. 2. The basic operation of the HMIPv6

\section{MAP Selection Based on the Traffic Characteristic}

\subsection{MAP Selection by Traffic Characteristic of MN}

A MN selects generally a MAP using the MAP option in Router Advertisement (RA) message received from MAP or AR. We propose a new scheme that Next MAPs (NMAPs) are predicted to reduce handover delay. The class of communication service is used as selection criteria for NMAP. The class of communication service is important to select the MAP. So, we consider the communication service that is used by MN. If MN uses realtime service, a rapid processing method should be prepared for realtime traffic processing during handover. Therefore, we should be consider what is the service using MN now. Also, we must choose MAP that can process 
rapidly the handover if handover is occurred. The traffic characteristics of MN are divided into realtime traffic such as multimedia traffic or voice traffic and data traffic such as best effort traffic. The characteristics of traffic are closely related to QoS of MN and also it should be an important element to choose MAP. It is shown as Table 1.

Table 1. Traffic characteristic of MN

\begin{tabular}{|l|l|}
\hline traffic characteristic & type \\
\hline \hline realtime traffic & streaming data traffic such as video or voice data traffic \\
\hline Non-realtime traffic & data traffic such as best effort traffic \\
\hline
\end{tabular}

Handover delay is more affected by handover between MAPs than handover between ARs because realtime traffic is susceptible to handover delay. When MN moves to another area, $\mathrm{MN}$ sends BU (Binding Update) message. BU requires approximately 1.5 round trip times between the $\mathrm{MN}$ and each $\mathrm{CN}$. In addition, one round-trip time is needed to update the HA; this can be done simultaneously while updating CNs. These round trip delays will disrupt active connections whenever a handoff to a new AR is performed. Moreover, in the case of wireless links, such a solution reduces the number of messages that sent to all CNs and the HA over the air interface. Accordingly, MAP selection by the distance is very important.

In Fig. 3, shows proposed system model.

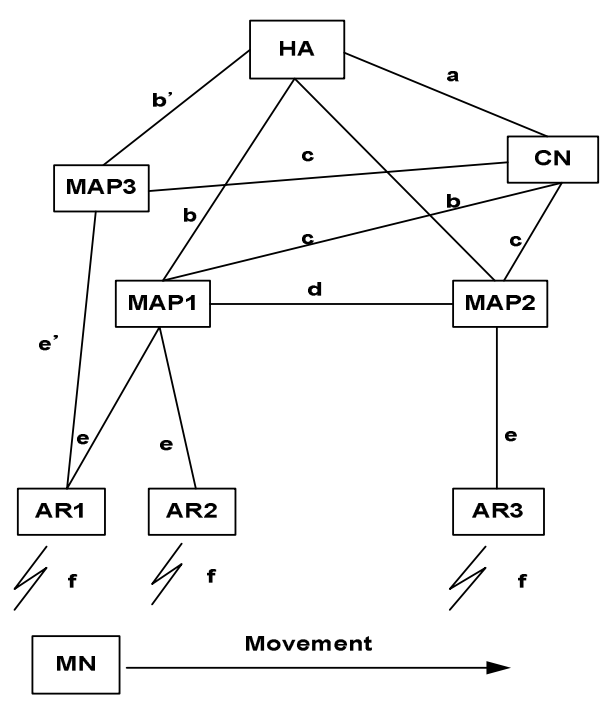

Fig. 3. System model of the proposed scheme 
In this paper, we propose a MAP selection scheme based on the traffic characteristic. If mobility frequency of $\mathrm{MN}$ is high, the speed of $\mathrm{MN}$ is very fast. However, if mobility frequency of $\mathrm{MN}$ is low, the speed of $\mathrm{MN}$ is slow relatively. As a result, if MN moves into one cell and cell resident time is short, then we may guess that $\mathrm{MN}$ moves very fast. If cell resident time is long, MN moves slow.

\section{Performance Evaluations}

\subsection{Mobility Model}

We assumed that there is hexagonal cellular network architecture, as shown in Fig. 4. Each MAP domain is assumed to consist of the different number of range rings, D. Rings of cells surround each cell as shown in Fig.4 [8]. Each ring $d(d>=0)$ is composed of $6 \mathrm{~d}$ cells. The innermost cell " 0 " is called the center cell. The cells labeled by 1 form the first ring around cell " 0 ", the cells labeled by 2 form the second ring around cell 0 and so forth. The number of cells $N$ (D) is calculated using the following equation:
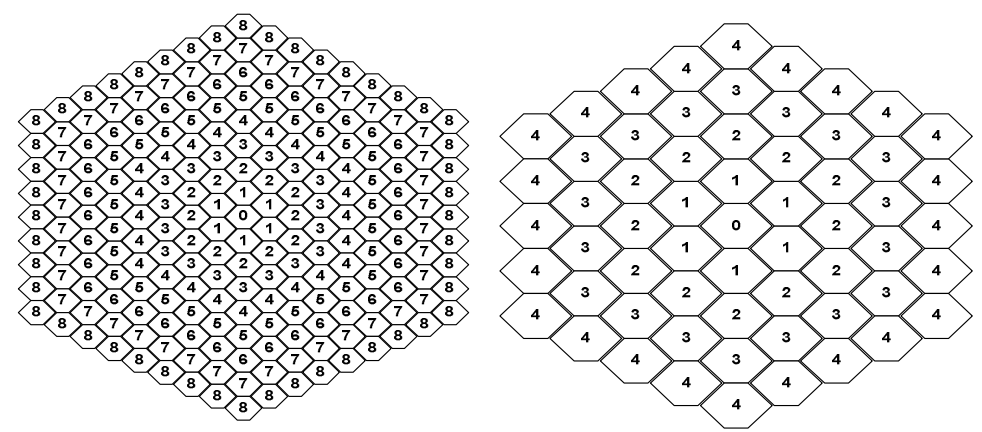

Fig. 4. Left is Hexagonal Cellular Network Architecture $(d=8)$ and Right is Hexagonal Cellular Network Architecture (d=4)

$$
N(D)=1+6 \cdot \sum_{d=1}^{D} d=1+3 \cdot D \cdot(D+1)
$$

Hexagonal Cellular Network Architecture $(\mathrm{d}=8)$ of Figure 4 shows the proposed hexagonal cellular network architecture of traffic characteristics.

The random-walk model is appropriate for pedestrian movements where mobility is generally confined to a limited geographical area such as residential and business buildings in the respect of user mobility model [9].

We consider the two-dimensional model used in Markov chain model in the respect of user mobility model [9]. In this model, the next position of an $\mathrm{MN}$ is equal to the previous position plus a random variable whose value is drawn independently from an arbitrary distribution. In addition, an $\mathrm{MN}$ moves to another cell area with a 
probability of 1-q and remains in the current cell with probability, q. If an $\mathrm{MN}$ is located in a cell of ring $\mathrm{d}(\mathrm{d}>0)$, the probability that a movement will result in an increase $(\mathrm{p}+(\mathrm{d}))$ or decrease $(\mathrm{p}-(\mathrm{d}))$ in distance from the center cell is given by

$$
P^{+}(d)=\frac{2 d+1}{6 d} \quad \text { and } \quad P^{-}(d)=\frac{2 d-1}{6 d}
$$

We define the state $\mathrm{k}$ of a Markov chain as the distance between the current cell of the MN and the center cell. This state is equivalent to the index of a ring in which the $\mathrm{MN}$ is located. As a result, the $\mathrm{MN}$ is said to be in state $\mathrm{k}$ if it is currently residing in ring d. The transition probabilities $\alpha_{d},{ }_{d+1}$ and $\beta_{d, d-1}$ represent the probabilities of the distance of the MN from the center cell increasing or decreasing, respectively. They are given as follow:

$$
\begin{gathered}
\alpha_{d, d+1}=\left\{\begin{array}{cc}
(1-q) & \text { if } \quad d=0 \\
(1-q) p^{+}(d) & \text { if } 1 \leq d \leq D
\end{array}\right. \\
\beta_{d, d-1}=(1-\mathrm{q}) \mathrm{p}^{-} \text {(d) }
\end{gathered}
$$

where $\mathrm{q}$ is the probability that an MN stays in the current cell.

We denote $\mathrm{p}_{\mathrm{d}, \mathrm{D}}$ as the steady-state probability of state $\mathrm{d}$ within a MAP domain consisting of D range rings. As Eq.(3) and Eq.(4), $P_{d, D}$ can be expressed in terms of the steady state probability $\mathrm{P}_{0, \mathrm{D}}$ as follows:

$$
P_{d, D}=P_{0, d} \prod_{i=0}^{d-1} \frac{\alpha_{i, i+1}}{\beta_{i+1, i}} \quad \text { for } 1 \leq \mathrm{d} \leq \mathrm{D}
$$

With the requirement $\sum_{d=0}^{D} P_{d, D}=1, \mathrm{P}_{\mathrm{d}, \mathrm{D}}$ can be expressed by

$$
P_{0, D}=\frac{1}{1+\sum_{d=1}^{D} \prod_{i=0}^{d-1} \frac{\alpha_{i, i+1}}{\beta_{i+1, i}}}
$$

where $\alpha_{d, d+1}$ and $\beta_{d, d-1}$ are obtained from Eq.(3) and Eq.(4) 


\subsection{Cost Functions}

In order to analyze the performance of wireless/mobile networks, the total cost, consisting of location update cost and paging cost, should be considered. However, since HMIPv6 [3] does not support paging functions, we divide the total cost into location update cost and packet delivery cost. In proposed scheme, we divide total cost into new location update and packet delivery cost. The location update cost, new location update and the packet delivery cost are denoted by $\mathrm{C}_{\text {location }}, \mathrm{C}_{\text {new-location }}$, and $\mathrm{C}_{\text {packet, }}$, respectively. Then, the total cost of HMIPv6 $\left(\mathrm{C}_{\text {total }}\right)$ and proposed scheme $\left(\mathrm{C}_{\text {new-total }}\right)$ can be obtained as follows:

$$
\begin{gathered}
C_{\text {total }}=C_{\text {location }}+C_{\text {packet }} \\
C_{\text {new-total }}=C_{\text {new-location }}+C_{\text {packet }}
\end{gathered}
$$

\subsubsection{Location Update Cost}

When a MN moves into a new MAP domain, it needs to configure two CoAs: an RCoA on the MAP's link and an on-link CoA(LCoA). In HMIPv6, an MN performs two types of binding update procedures: the global binding update and the local binding update. In global binding update, an MN registers its RCoA with the CNs and the HA. On the other hand, if an MN changes its current address within a local MAP domain, it only needs to register to this registration. $\mathrm{C}_{\mathrm{g}}, \mathrm{C}_{\mathrm{new}-\mathrm{g}}$ and $\mathrm{C}_{\mathrm{l}}$ denote the signaling costs in the global binding update, the global binding update of proposed scheme and the local binding update, respectively. In the IP networks, the signaling cost is proportional to the distance between two network entities. $\mathrm{C}_{\mathrm{g}}, \mathrm{C}_{\mathrm{new}-\mathrm{g}}$, and $\mathrm{C}_{\mathrm{l}}$ can be obtained from the below equations.

$$
\begin{gathered}
C_{g}=2 \cdot(k \cdot f+\tau \cdot(b+e))+2 \cdot N_{C N} \cdot(k \cdot f+\tau \cdot(b+c)) \\
+P C_{H A}+N_{C N} \cdot P C_{C N}+P C_{M A P} \\
C_{\text {new }-g}=2 \cdot\left(k \cdot f+\tau \cdot\left(b^{\prime}+e^{\prime}\right)\right)+2 \cdot N_{C N} \cdot\left(k \cdot f+\tau \cdot\left(b^{\prime}+c\right)\right) \\
+P C_{H A}+N_{C N} \cdot P C_{C N}+P C_{M A P} \\
C_{l}=2 \cdot\left(k \cdot f+\tau \cdot e^{\prime}\right)+P C_{M A P}
\end{gathered}
$$

Here $\tau$ and $\mathrm{k}$ are the unit transmission costs in a wired and a wireless link, respectively. $\mathrm{PC}_{\mathrm{HA}}, \mathrm{PC}_{\mathrm{CN}}$ and $\mathrm{PC}_{\mathrm{MAP}}$ are the processing costs for binding update procedures at the $\mathrm{HA}$, the $\mathrm{CN}$ and the MAP, respectively. Let b, b', c, e, e' and $\mathrm{f}$ be 
the hop distance between nodes. $\mathrm{N}_{\mathrm{CN}}$ denotes the number of $\mathrm{CNs}$ which are communicating with the $\mathrm{MN}$.

HMIPv6 is an enhanced Mobile IPv6 to minimize the signaling cost using a local agent called MAP. The MAP can be located at any level in a hierarchical network of routers, including the AR. The MAP in HMIPv6 treats the mobility management inside a domain. Thus, when a MN moves around the sub-networks within a single domain, the MN sends a BU message only to the current MAP. In proposed scheme, we reduce the probability of the global binding update.

In terms of the random walk mobility model, the probability that a $\mathrm{MN}$ performs a global binding update is as follows:

$$
P_{D, D} \cdot \alpha_{d, d+1}
$$

Specifically, if a MN is located in range ring $\mathrm{D}$, the boundary ring of a MAP domain is composed of $\mathrm{D}$ range rings, and performs a movement from range ring $\mathrm{D}$ to range ring $\mathrm{D}+1$. The $\mathrm{MN}$ then performs the global binding update procedure. In other cases, except this movement, the $\mathrm{MN}$ only performs a local binding update procedure. Hence, the location update cost of normal and proposed scheme per unit time can be expressed as follows:

$$
\begin{aligned}
C_{\text {location }} & =\frac{P_{D, D} \cdot \alpha_{D, D+1} \cdot C_{g}+\left(1-P_{D, D} \cdot \alpha_{D, D+1}\right) \cdot C_{l}}{T} \\
C_{\text {new -location }} & =\frac{P_{D, D} \cdot \alpha_{D, D+1} \cdot C_{n e w-g}+\left(1-P_{D, D} \cdot \alpha_{D, D+1}\right) \cdot C_{l}}{T}
\end{aligned}
$$

where $\mathrm{T}$ is the average cell residence time.

\subsubsection{Packet Delivery Cost}

The packet delivery cost, $\mathrm{C}_{\text {packet }}$, in HMIPv6 can then be calculated as follows:

$$
C_{\text {PACKET }}=C_{M A P}+C_{H A}+C_{C N-M N}
$$

In $\mathrm{Eq}(15), \mathrm{C}_{\mathrm{MAP}}$ and $\mathrm{C}_{\mathrm{HA}}$ denote the processing costs for packet delivery at the MAP and the HA, respectively. $\mathrm{C}_{\mathrm{CN}-\mathrm{MN}}$ denotes the packet transmission cost from the $\mathrm{CN}$ to the $\mathrm{MN}$.

In HMIPv6, a MAP maintains a mapping table for translation between RCoA and LCoA. The mapping table is similar to that of the HA, and it is used to track the current locations (LCoA) of the MNs. All packets directed to the MN will be received 
by the MAP and tunneled to the MN's LCoA using the mapping table. Therefore, the lookup time required for the mapping table also needs to be considered. Specifically, when a packet arrives at the MAP, the MAP selects the current LCoA of the destination $\mathrm{MN}$ from the mapping table and the packet is then routed to the $\mathrm{MN}$. Therefore, the processing cost at the MAP is divided into the lookup cost $\left(\mathrm{C}_{\text {lookup }}\right)$ and the routing cost $\left(\mathrm{C}_{\text {routing }}\right)$. The lookup cost is proportional to the size of the mapping table. The size of the mapping table is proportional to the number of MNs located in the coverage of a MAP domain [10]. On the other hand, the routing cost is proportional to the logarithm of the number of ARs belonging to a particular MAP domain [4]. Therefore, the processing cost at the MAP can be expressed as Eq. (17). In Eq.(17), $\lambda_{s}$ denotes the session arrival rate and $S$ denotes the average session size in the unit of packet. $\alpha$ and $\beta$ are the weighting factors. Let $\mathrm{N}_{\mathrm{MN}}$ be the total number of users located in a MAP domain. This paper assumes that the average number of users located in the coverage of an AR is $\mathrm{K}$. Therefore, the total number of users can be obtained using Eq. (16).

$$
\begin{gathered}
N_{M N}=N_{A R} \times K \\
C_{M A P}=\lambda_{s} \cdot \bar{S} \cdot\left(C_{\text {lookup }}+C_{\text {routing }}\right) \\
=\lambda_{s} \cdot \bar{S} \cdot\left(\alpha N_{M N}+\beta \log \left(N_{A R}\right)\right)
\end{gathered}
$$

In MIPv6, the route optimization is used to resolve the triangular routing problem. Therefore, the only first packet of a session transits the HA to detect whether an MN moves into foreign networks or not. Subsequently, all successive packets of the session are directly routed to the MN. The processing cost at the HA can be calculated as follows:

$$
C_{H A}=\lambda_{S} \cdot \theta_{H A}
$$

where $\Theta_{\text {HA }}$ refers to a unit packet processing cost at the HA.

Since HMIPv6 supports the route optimization, the transmission cost in HMIPv6 can be obtained using Eq. (19). As mentioned before, $\tau$ and $\kappa$ denote the unit transmission costs in a wired and a wireless link, respectively.

$$
C_{C N-M N}=\tau \cdot \lambda_{s} \cdot((S-1) \cdot(c+e)+(a+b+e))+k \cdot \lambda_{s} \cdot S
$$




\section{Numerical Results}

In this section, we provide some numerical evaluation to demonstrate the performance of proposed scheme as compared with normal HMIPv6. The parameter values for the analysis were referenced from [10], [11] and [12]. They are shown in Table 2.

Table 2. Numerical simulation parameter for performance analysis

\begin{tabular}{|c|c|c|c|c|c|}
\hline parameter & value & parameter & value & parameter & value \\
\hline$\alpha$ & 0.1 & $\mathrm{a}$ & 6 & $\mathrm{~b}$ & 3 \\
\hline$\beta$ & 0.2 & $\mathrm{~b}$ & 6 & $\mathrm{e}$ & 4 \\
\hline$\gamma$ & 0.05 & $\mathrm{c}$ & 4 & $\mathrm{~N}_{\mathrm{CN}}$ & 2 \\
\hline$\theta_{H A}$ & 20 & $\mathrm{~d}$ & 1 & $\mathrm{PC}_{\mathrm{HA}}$ & 24 \\
\hline$\tau$ & 1 & $\mathrm{e}$ & 2 & $\mathrm{PC}_{\mathrm{MAP}}$ & 12 \\
\hline$k$ & 2 & $\mathrm{f}$ & 1 & $\mathrm{PC}_{\mathrm{CN}}$ & 6 \\
\hline
\end{tabular}

Fig 5 shows the variation in the location update cost as the average cell residence time is changed in the random-walk model. The location updates cost becomes less as the average cell residence time increases. In a comparison of proposed scheme with HMIPv6, proposed scheme reduces the location update cost by 5\% approximately.

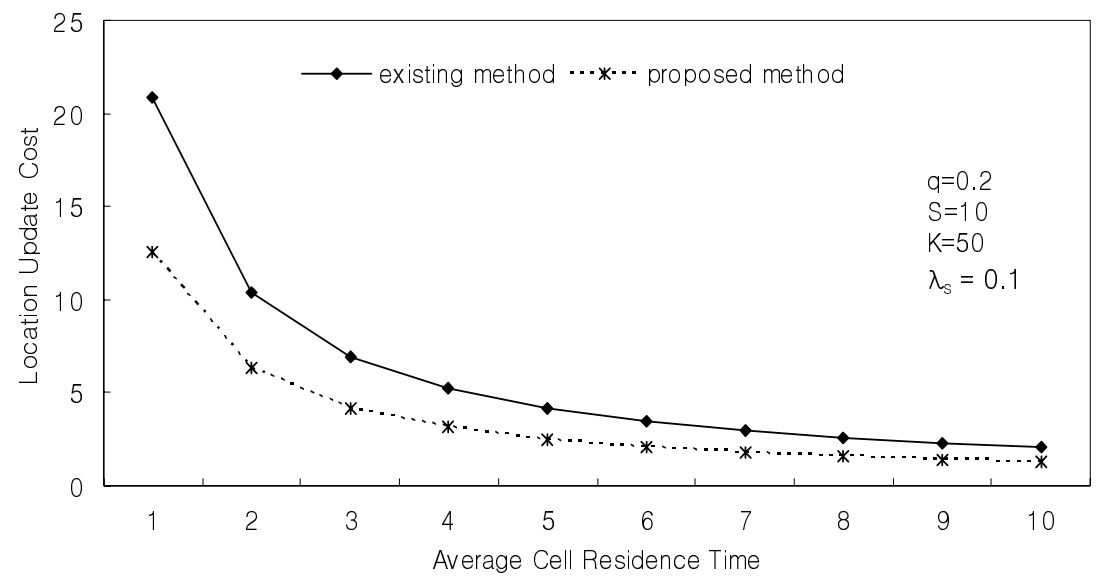

Fig. 5. Location update cost as function of average cell residence time of $\mathrm{MN}$

Fig 6 shows the total cost of average cell residence time in random-walk model.

When the average cell residence time of $\mathrm{MN}$ is below 5 seconds, in the other words, MN moves frequently, $\mathrm{MN}$ is superior in respect of transmission ability of 


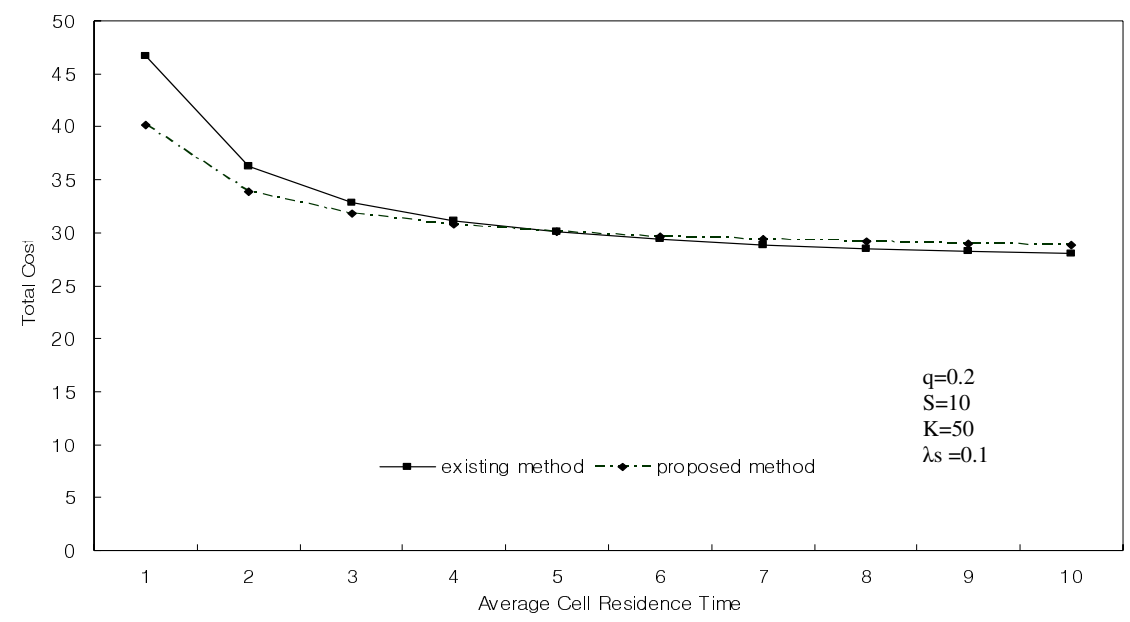

Fig. 6. Total cost as function of average cell residence time of $\mathrm{MN}$

realtime traffic. As a result, if the cell residence time of $\mathrm{MN}$ is below 5 seconds, proposed method is preferred. However, if the cell residence time of $\mathrm{MN}$ is over 5 seconds, conventional method is preferred.

\section{Conclusion}

The MAP, which is proposed in HMIPv6, is proposed for mobility management of MNs. But, the connection requests are concentrated to the upper level MAPs. The concentration on specific MAP cannot guarantee the stable service for handover transaction according to overload and the failure on the selection of specific MAP leads to additional handover delay. Therefore, this paper proposed a scheme, which prevents concentration to the specific MAP by distributing connection requests to various MAPs based on the characteristic of mobile devices.

The proposed scheme reduces the location update cost by $5 \%$ and the total cost by $34 \%$ approximately.

\section{References}

1. D. Johnson, C. Perkins and J. Arrko, "Mobility support in IPv6," Internet Draft, IETF, draft-ietf-mobileip-ipv6-20.txt(work in progress), Jan. 2003.

2. J. Xie and F. Akyildiz, "A novel distributed dynamic location management scheme for minimizing signaling costs in Mobile IP," IEEE Trans. on Mobile Computing, Vol. 1, No, 3, pp. 163-175, Sep. 2002.

3. H. Soliman, C. Castelluccia, K. E. Malki and L. Bellier, "Hierarchical MIPv6 (HMIPv6) mobility management," Internet Draft, Nov. 2001.

4. Sangheon Pack, Byoungwook Lee, and Yanghee Choi, "Load Control Scheme at Local Mobility Agent in Mobile IPv6 Networks," WWC04, May. 2004. 
5. S. Deering and B. Hinden, "Internet Protocol version6 (IPv6) specification," IETF, RFC2460, Dec. 1998.

6. P. Reinbold and O. Bonaventure, "A Comparison of IP Mobility Protocol," Tech. Rep. Infonet-TR-2001-07, University of Namur, Infonet Group, Jun. 2001.

7. Abbas Jamalipour, The Wireless Mobile Internet, Wiley, 2003.

8. J.S.M. Ho and I. F. Akyildiz, "Mobile user location update and paging under delay constrainsts," ACM-Baltzer J. Wireless Networks, vol. 1, pp. 413-425, Dec. 1995.

9. I.F. Akyildiz and W. Wang, "A dynamic location management scheme for next-generation multitier PCS systems," IEEE Trans. Wireless Commun., vol.1, no.1, pp.178-189, Jan. 2002.

10. Sangheon Pack and Yanghee Choi, "A Study on performance of hierarchical mobile IPv6 in IP-based cellular networks," IEICE Transactions on Communications, vol. E87-B no. 3 pp.462-469, Mar. 2004.

11. M. Woo, "Performance analysis of mobile IP regional registration," IEICE Trans. Commun., vol.E86-B, no.2, pp.472-478, Feb. 2003.

12. X. Zhang, J. G. Castellanos, and A. T. Capbell, "P-MIP: Paging extensions for mobile IP," ACM Mobile Networks and Applications, vol.7, no.2, pp.127-141, 2002. 